\title{
FOXK1 plays an oncogenic role in the progression of hilar cholangiocarcinoma
}

\author{
YUJIE FENG ${ }^{1,2}$, ZHIGANG BAI $^{1}$, JIANNING SONG ${ }^{1}$ and ZHONGTAO ZHANG ${ }^{1}$ \\ ${ }^{1}$ Department of General Surgery, Beijing Friendship Hospital, Capital Medical University, \\ Beijing Key Laboratory of Cancer Invasion and Metastasis Research and National Clinical Research Center \\ for Digestive Diseases, Beijing 100050; ${ }^{2}$ Department of Hepatobiliary and Pancreatic Surgery, \\ The Affiliated Hospital of Qingdao University, Qingdao, Shandong 266000, P.R. China
}

Received July 22, 2019; Accepted November 3, 2020

DOI: $10.3892 / \mathrm{mmr} .2020 .11730$

\begin{abstract}
Hilar cholangiocarcinoma (HC) has a poor outcome in terms of survival. Forkhead box K1 (FOXK1) dysregulation is critical in solid tumors, which serves a pivotal role in the biological characteristics, such as invasion and migration, but its expression and functions in $\mathrm{HC}$ are unclear. The present study investigated the clinical significance and biological functions of FOXK1 in HC. Tumor microarrays and immunohistochemistry were used to evaluate FOXK1 in $\mathrm{HC}$ and its expression was modulated to determine its effects on chemoresistance and tumorigenesis. FOXK1 was highly expressed in $\mathrm{HC}$ and cell lines, which was associated with tumor invasion, regional lymph node metastasis, tumor recurrence and poor prognosis. Silencing FOXK1 in HC cells inhibited invasion and migration, upregulated E-cadherin, and downregulated vimentin, matrix metallopeptidase 9 and Twist in HC cells. Sensitivity to 5-fluorouracil and cisplatin was increased, and glutathione $\mathrm{S}$-transferase $\pi$, multidrug resistance mutation 1 and P-glycoprotein expression levels were downregulated in RBE cells in vitro following FOXK1 knockdown. These results indicated that FOXK1 plays an oncogenic role in HC progression and can serve as a novel therapeutic target for HC.
\end{abstract}

\section{Introduction}

Hilar cholangiocarcinomas (HCs) are the most common type of cholangiocarcinoma and are highly prevalent in Southeast Asia, although uncommon in the USA (1). The only strategy

Correspondence to: Professor Zhongtao Zhang, Department of General Surgery, Beijing Friendship Hospital, Capital Medical University, Beijing Key Laboratory of Cancer Invasion and Metastasis Research and National Clinical Research Center for Digestive Diseases, 95 Yongan Road, Beijing 100050, P.R. China E-mail: zhangzht@medmail.com.cn

Key words: chemotherapeutic sensitivity, epithelial-mesenchymal transition, forkhead box K1, hilar cholangiocarcinoma, tumor invasion for treating patients and prolonging overall survival (OS) is complete resection with negative surgical margins (2). However, in most patients, the tumors are unresectable due to locally advanced or metastatic disease at diagnosis (3). Recently, advances in revealing the genetic landscape of HC have led to the identification of several promising systemic therapeutic agents or strategies that could improve the outcome of patients with $\mathrm{HC}$ (4).

Forkhead box K1 (FOXK1) is a member of the FOX transcription factor family, which plays critical roles in embryonic development and organogenesis, and regulates a variety of physiological processes, including metabolism, cell signaling and cell proliferation (5-7). The dysregulation of FOXK1 expression and subcellular localization leads to the uncontrolled development and progression of human solid cancer types. For instance, increasing evidence has demonstrated that FOXK1 knockdown can inhibit cell proliferation, migration and invasion in prostate cancer (8), hepatocellular carcinoma (9) and esophageal cancer (10), whereas its enhanced expression facilitates cell proliferation and metastasis in ovarian (11) and esophageal cancer (10). These studies suggest that FOXK1 has a role in tumorigenesis. However, its expression pattern and function in $\mathrm{HC}$ are not well defined.

The present study investigated the clinical significance and biological functions of FOXK1 in HC. The results demonstrated that FOXK1 was upregulated in $\mathrm{HC}$ tissues and that its high expression was significantly associated with neural invasion and lymph node (LN) metastasis. In addition, the nuclear expression of FOXK1 was found to be associated with recurrence and poor outcome in patients with HC. FOXK1 knockdown in vitro inhibited the proliferation and migration of $\mathrm{HC}$ cells. To the best of the authors' knowledge, the present study was the first to reveal the expression profile of FOXK1 and its function in $\mathrm{HC}$ cell proliferation and metastasis, highlighting its potential as a therapeutic target for this disease.

\section{Materials and methods}

Patient samples and cell culture. Tissue microarrays (TMAs) of 48 resected HC specimens and 15 matched non-cancerous bile duct tissues (with $>5-\mathrm{mm}$ distance from the primary tumor's edge) obtained from the Eastern Hepatobiliary Hospital 
and Changhai Hospital (Shanghai, China) were constructed as previously described (12). Of the patients, 31 were men and 17 women, with a mean age of 62 years (range, $42-78$ years). Patient characteristics are given in Table I. The median followup duration was 16 months (range, 1-59 months). The tissue sample experiments were approved by the Ethics Committee of The Affiliated Hospital of Qingdao University. Informed consent written from all participants (or their parent or legal guardian in the case of children under 16) was obtained to participate in the study or to use their tissues. Human cholangiocarcinoma cell lines (FRH-0201, QBC939 and RBE) were obtained from The Cell Bank of Type Culture Collection of the Chinese Academy of Sciences and. The expression of FOXK1 in the four cell lines were detected by western blotting, and it was identified that RBE cells exhibited the highest expression, so the RBE cell line was selected as the experimental cells. These cells were maintained in Dulbecco's modified Eagle's medium (Invitrogen; Thermo Fisher Scientific, Inc.) with $10 \%$ fetal bovine serum (Invitrogen; Thermo Fisher Scientific, Inc.) and cultured at $37^{\circ} \mathrm{C}$ with $5 \% \mathrm{CO}_{2}(12)$.

Immunohistochemistry and evaluation of HC specimens. Sections $(4 \mu \mathrm{m})$ of TMAs were prepared and processed for the immunohistochemical analysis of FOXK1 (1:100; cat. no. ab18196; Abcam), which was carried out according to a previous study (13). A streptavidin-biotin kit (cat. no. KIT-9720; Fuzhou Maixin Biotech Co., Ltd.) was used to visualize antibody binding in these sections. Immunostaining of FOXK1 was evaluated by two individuals (ZGB and YJF) and a semiquantitative scoring system was used in the present study, as previously reported (12). A weighted score was generated for each case ranging from 0 ( $0 \%$ of cells stained) to $300(100 \%$ of cells stained at $>3$ intensity) as previous study described, a score of $<75$ was defined as low expression and that of $\geq 75$ was defined as high expression (12).

Cell transfection. FOXK1 short hairpin (sh)RNA (cat. no. PR6021) and scrambled shRNA plasmids (pLent-U6GFP-Puro) were constructed by Shanghai GeneChem Co., Ltd. Next, $1 \times 10^{5}$ RBE cells were seeded in 6-well plates and transfected with FOXK1 shRNA (shFOXK1) or scrambled shRNA (shNC) at $5 \mathrm{ng}$ shRNA plasmid per well using Lipofectamine ${ }^{\circledR}$ 2000 reagent (Invitrogen; Thermo Fisher Scientific, Inc.), according to the manufacturer's instructions. These cells were then subcultured and selected in the presence of puromycin $(1 \mu \mathrm{g} / \mathrm{ml})$ for 3 days between transfection and experimentation at $37^{\circ} \mathrm{C}$ to generate stable NC and FOXK1-knockdown cells.

Cell Counting Kit-8 (CCK-8) analysis. After obtaining stable FOXK1-knockdown or NC RBE lines, 5,000 cells per well were seeded in 96-well plates. At the indicated times $(24,48$ and $72 \mathrm{~h}$ ), the CCK-8 assay (Dojindo Molecular Technologies, Inc.) was performed to assess the results. This experiment was performed in triplicate.

Cell viability assay. FOXK1-knockdown and NC RBE cells were seeded in 96-well plates and treated with different concentrations $(0,0.25,0.5,1,2.5,5,10,25,50$ and $100 \mu \mathrm{g} / \mathrm{ml})$ of 5-FU and cisplatin (DDP) when the cell density reached $60-70 \%$. Cell viability was assayed using an MTT assay
Table I. Association between the expression of FOXK1 and clinicopathological parameters of hilar cholangiocarcinoma.

\begin{tabular}{lrrr}
\hline $\begin{array}{l}\text { Clinicopathological } \\
\text { parameters }\end{array}$ & $\mathrm{N}$ & FOXK1,n $(\%)$ & P-value \\
\hline $\begin{array}{l}\text { Tumor size, cm } \\
\leq 3\end{array}$ & 17 & $8(47.1)$ & 0.135 \\
3 & 31 & $23(74.2)$ & \\
Nerve invasion & & & 0.838 \\
$\quad$ Yes & 25 & $17(68.0)$ & \\
$\quad$ No & 23 & $15(65.2)$ & \\
T stage & & & 0.005 \\
T1-3 & 6 & $1(16.7)$ & \\
T4 & 42 & $31(73.8)$ & \\
N stage & & & 0.008 \\
N0 & 15 & $6(40.0)$ & \\
$\quad$ N1-2 & 33 & $26(78.8)$ & \\
Differentiation & & & 0.480 \\
High/moderate & 36 & $23(63.9)$ & \\
Low/undifferentiated & 12 & $9(75.0)$ & \\
TNM & & & 0.095 \\
I/II & 19 & $10(52.6)$ & \\
III/IV & 29 & $22(75.9)$ & \\
\end{tabular}

FOXK1, forkhead box K1; T, tumor; N, node; M, metastasis.

(Dojindo Molecular Technologies, Inc.) according to the manufacturer's instructions.

Transwell invasion assay. Transwell chambers $(8 \mu \mathrm{m}$ pore size; Corning, Inc.) were used to assess the effect of FOXK1 knockdown on cell invasion. After stable transfection with shFOXK1 or shNC, 10,000 cells were resuspended in serumfree DMEM and placed in the upper well of the chamber coated with $25 \mu \mathrm{g}$ Matrigel (BD Biosciences) for the invasion assay. The lower well was filled with DMEM containing $10 \%$ FBS. The chambers were maintained at $37^{\circ} \mathrm{C}$ for $24 \mathrm{~h}$, and then removed. The cells on the upper surface of the chambers were removed using a cotton swab, while the cells on the lower surface were stained with $0.1 \%$ crystal violet for $30 \mathrm{~min}$ at room temperature and counted in five representative (magnification, x200) fields per insert under an Inversion Microscope (Zeiss AG) by two individuals (YJF and ZGB), who were blinded to the study.

Wound healing assay. The indicated cells were cultured in 6-well plates in monolayers and were pre-incubated with Mitomycin-C $(10 \mu \mathrm{g} / \mathrm{ml})$ for $1 \mathrm{~h}$ at $37^{\circ} \mathrm{C}$ to suppress cell proliferation. Then, cells were plated in serum-starved medium. A sterile 200- $\mu 1$ pipette tip was used to create wounds and the areas of the wound fields were observed and images (magnification, $\mathrm{x} 200$ ) were taken at $0,24,48$ and $72 \mathrm{~h}$ following wound creation using an inverted microscope (Carl Zeiss AG).

Western blotting. Total protein was extracted from the cells. Western blotting was performed as previously described (14). 
A

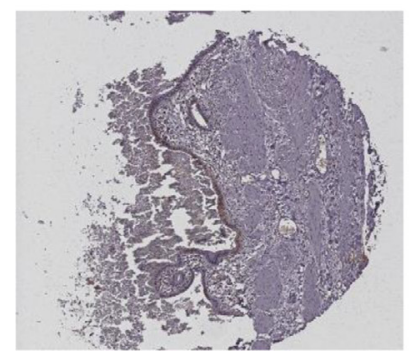

B

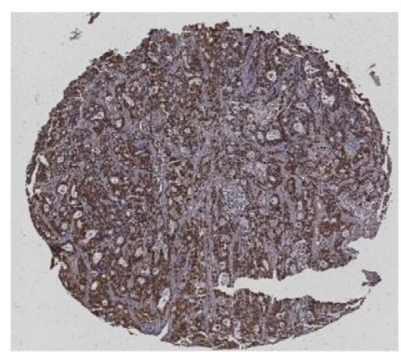

C

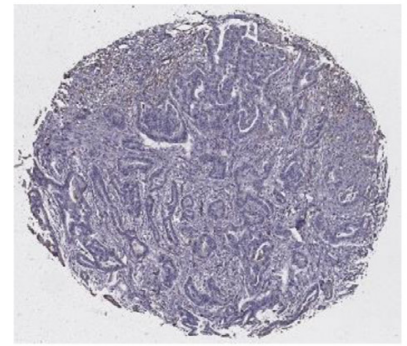

D

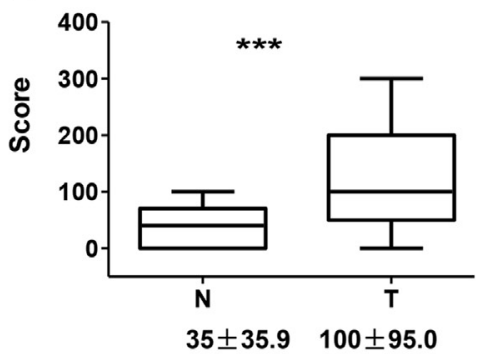

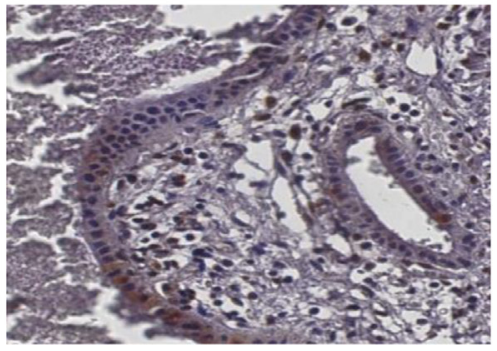
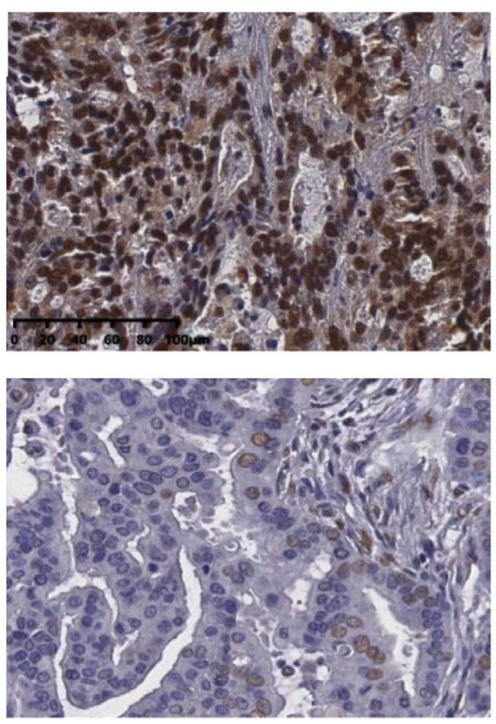

$\mathbf{E}$

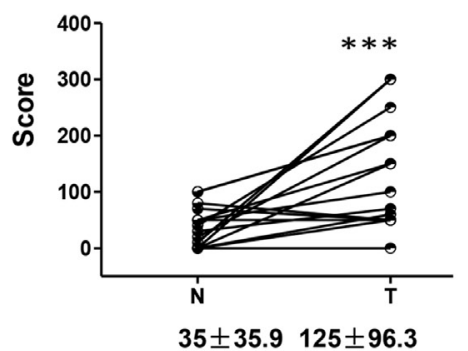

Figure 1. FOXK1 expression in tumor tissues and non-cancerous bile duct epithelium. (A) Low expression of FOXK1 in non-cancerous bile duct tissues; (B) high expression of FOXK1 in representative tumor tissues; (C) low expression of FOXK1 in representative tumor tissues. Left panel magnification, x40; right panel magnification, $\mathrm{x} 200$. (D) The positive average score of all cases of FOXK1 staining in the $\mathrm{N}$ group was lower than that of the T group. (E) For the 15 matched cases, the positive average score of FOXK1 staining in $\mathrm{N}$ was also lower than $\mathrm{T}$. ${ }^{* * *} \mathrm{P}<0.001$ vs. T group. FOXK1, forkhead box K1; $\mathrm{N}$, noncancerous tissues; $\mathrm{T}$, tumor samples.

All antibodies used are as follows: Anti-FOXK1 (1:1,000; cat. no. ab18196; Abcam), anti-matrix metallopeptidase (MMP)-9 (1:500; cat. no. ab228402; Abcam), anti-Twist (1:1,000; cat. no. ab50581; Abcam), anti-E-cadherin (1:1,000; cat. no. 3195; Cell Signaling Technology, Inc.), anti-vimentin (1:1,000; cat. no. 5741; Cell Signaling Technology, Inc.), antiglutathione S-transferase (GST)- $\pi$ (1:500; cat. no. 66001-2-Ig; ProteinTech Group, Inc.), anti-MDR-1 (1:200; cat. no. sc13131; anti-Santa Cruz Biotechnology, Inc.), anti-P-glycoprotein (P-gp; 1:500; cat. no. ab170904; Abcam), and anti-GAPDH (1:1,000; cat. no. 5174; Cell Signaling Technology, Inc.). The signals were detected using horseradish peroxidase-based chemiluminescence analysis.

Statistical analyses. Statistical analyses were carried out using SPSS statistical software (version 16.0; SPSS, Inc.) and Prism software (version 5.0; GraphPad Software, Inc.).
Data are presented as the mean \pm standard error of the mean. The differences between two groups were evaluated with a Student's t-test. Categorical data were analyzed using $\chi^{2}$ tests. The Kaplan-Meier log-rank method was used to estimate survival rates, and the Cox proportional hazards model for multivariate survival analysis was used to assess predictors related to recurrence or survival. $\mathrm{P}<0.05$ was considered to indicate a statistically significant difference.

\section{Results}

Highexpression ofFOXK1 inpatientswithHC.Immunostaining revealed that FOXK1 was primarily localized to the nucleus of the normal bile duct epithelium and cancer cells (Fig. 1). The epithelium in the adjacent non-cancerous tissues showed low expression of FOXK1, with an average score of $35 \pm 35.9$ (Fig. 1A and D). Furthermore, 32 cases (66.7\%) showed high 
Table II. Univariate and multivariate analysis of variables associated with disease-free survival in patients with hilar cholangiocarcinoma.

\begin{tabular}{|c|c|c|c|c|c|c|}
\hline \multirow[b]{2}{*}{ Variables } & \multirow[b]{2}{*}{$\mathrm{N}$} & \multirow[b]{2}{*}{ DFS, months } & \multicolumn{2}{|c|}{ P-value } & \multirow[b]{2}{*}{ HR } & \multirow[b]{2}{*}{$95 \%$ CI } \\
\hline & & & Univariate analysis & Multivariate analysis & & \\
\hline Tumor invasion & & & 0.006 & 0.065 & 0.146 & $0.019-1.125$ \\
\hline T1-T3 & 6 & 51.2 & & & & \\
\hline $\mathrm{T} 4$ & 42 & 16.0 & & & & \\
\hline Regional LN positive & & & 0.05 & 0.423 & 0.690 & $0.279-1.710$ \\
\hline No & 15 & 37.0 & & & & \\
\hline Yes & 33 & 15.0 & & & & \\
\hline FOXK1 & & & $<0.001$ & 0.014 & 0.253 & $0.084-0.759$ \\
\hline Low & 16 & 42 & & & & \\
\hline High & 32 & 11 & & & & \\
\hline
\end{tabular}

HR, hazard ratio; CI, confidence interval; FOXK1, forkhead box K1; LN, lymph node.

Table III. Univariate and multivariate analysis of variables associated with OS in patients with hilar cholangiocarcinoma.

\begin{tabular}{|c|c|c|c|c|c|c|}
\hline \multirow[b]{2}{*}{ Variables } & \multirow[b]{2}{*}{$\mathrm{N}$} & \multirow[b]{2}{*}{ DFS, months } & \multicolumn{2}{|c|}{ P-value } & \multirow[b]{2}{*}{ HR } & \multirow[b]{2}{*}{$95 \% \mathrm{CI}$} \\
\hline & & & Univariate analysis & Multivariate analysis & & \\
\hline Tumor invasion & & & 0.014 & 0.114 & 0.297 & $0.066-1.340$ \\
\hline T1-T3 & 6 & 50 & & & & \\
\hline $\mathrm{T} 4$ & 42 & 16 & & & & \\
\hline Regional LN positive & & & 0.033 & 0.390 & 0.678 & $0.279-1.646$ \\
\hline No & 15 & 42 & & & & \\
\hline Yes & 33 & 14 & & & & \\
\hline FOXK1 & & & $<0.001$ & 0.037 & 0.357 & $0.136-0.940$ \\
\hline Low & 16 & 50 & & & & \\
\hline High & 32 & 14 & & & & \\
\hline
\end{tabular}

HR, hazard ratio; CI, confidence interval; FOXK1, forkhead box K1; OS, overall survival; LN, lymph node.

expression (Fig. 1B) and the other 16 cases presented low expression (Fig. 1C). The average score for FOXK1 in HC was $100 \pm 95.0$, which was significantly higher than that in the non-cancerous bile duct epithelium $(\mathrm{P}=0.0003)$. Similarly, in the matched cases with both $\mathrm{HC}$ and adjacent non-cancerous epithelium, the score was $125 \pm 96.3$ and $35 \pm 35.9$, respectively $(\mathrm{P}=0.0006$; Fig. 1E). In one intracholangial papillary tumor (Fig. 2), $50 \%$ of the cells on the surface showed FOXK1 expression (Fig. 2A), whereas the tumor cells at the front edge of the invasive front showed $100 \%$ FOXK1 expression (Fig. 2B and C), indicating that FOXK1 might be involved in the progression of $\mathrm{HC}$.

Association between high FOXK1 expression and clinicopathological variables of $H C$. Table I summarizes the association between high FOXK1 expression and clinicopathological variables of HC. A statistically significant association was observed between high FOXK1 expression and tumor invasion and regional LN metastasis. Furthermore, high FOXK1 expression occurred more frequently in highly invasive tumors (invasion level T4, 73.8\%) than in less invasive tumors (levels T1-T3, 16.7\%; $\mathrm{P}=0.005)$. With regard to the $\mathrm{N}$ stage, FOXK1 was highly expressed in $\mathrm{HCs}$ with regional LN metastasis (78.8\%) compared with that in HCs without LN metastasis (40\%) $(\mathrm{P}=0.008)$. This indicated that the expression of FOXK1 is associated with the prognosis of $\mathrm{HC}$.

Relationship between high FOXK1 expression and HC tumor recurrence or outcome. For this assessment, the cohort consisted of 34 male $(70.8 \%)$ and 14 female (29.2\%) patients with a median age of 55 years (range, $31-79$ years). The median disease-free survival (DFS) in patients with resected $\mathrm{HC}$ was 14 months. Patients with tumors exhibiting high FOXK1 expression had a significantly shorter DFS than that in patients with low FOXK1 expression (11 vs. 42 months; $\mathrm{P}<0.001$; Fig. 3A). Additionally, it was noted that tumor invasion $(\mathrm{P}=0.006)$ and regional $\mathrm{LN}$ metastasis $(\mathrm{P}=0.05)$ significantly affected DFS based on the univariate analysis. In contrast to the univariate 

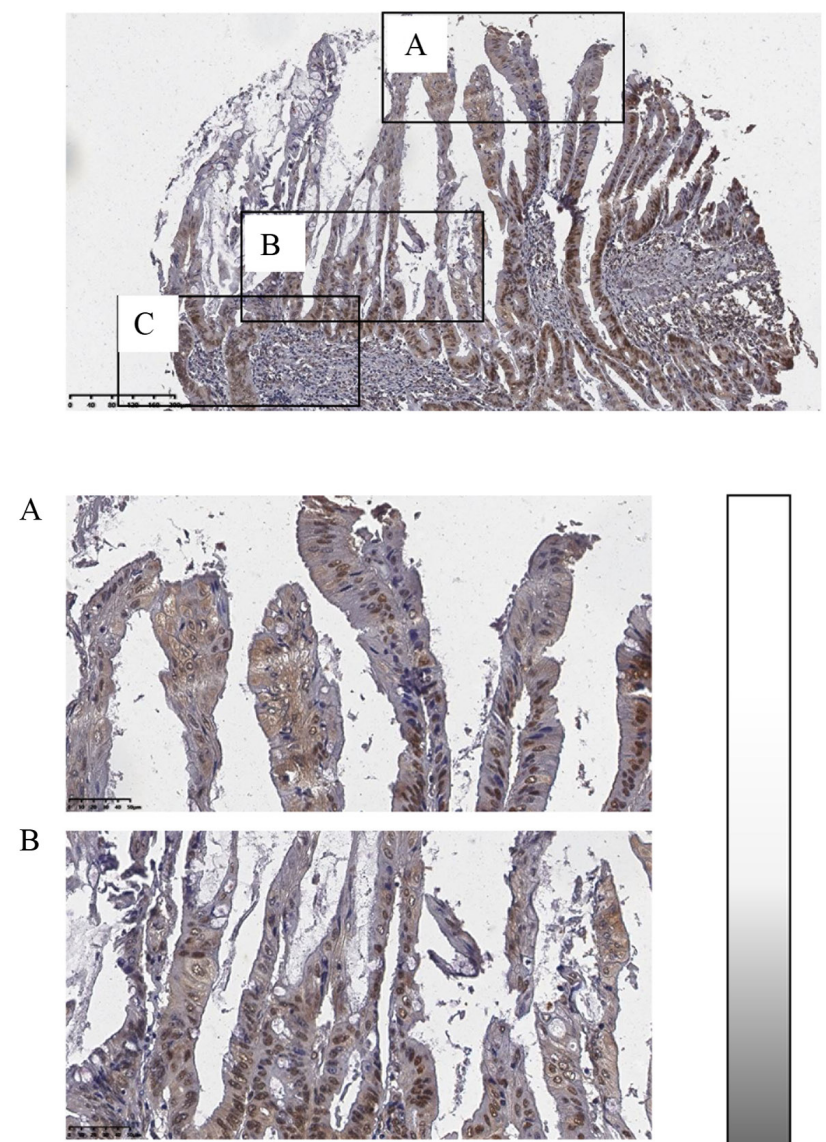

$\mathrm{C}$
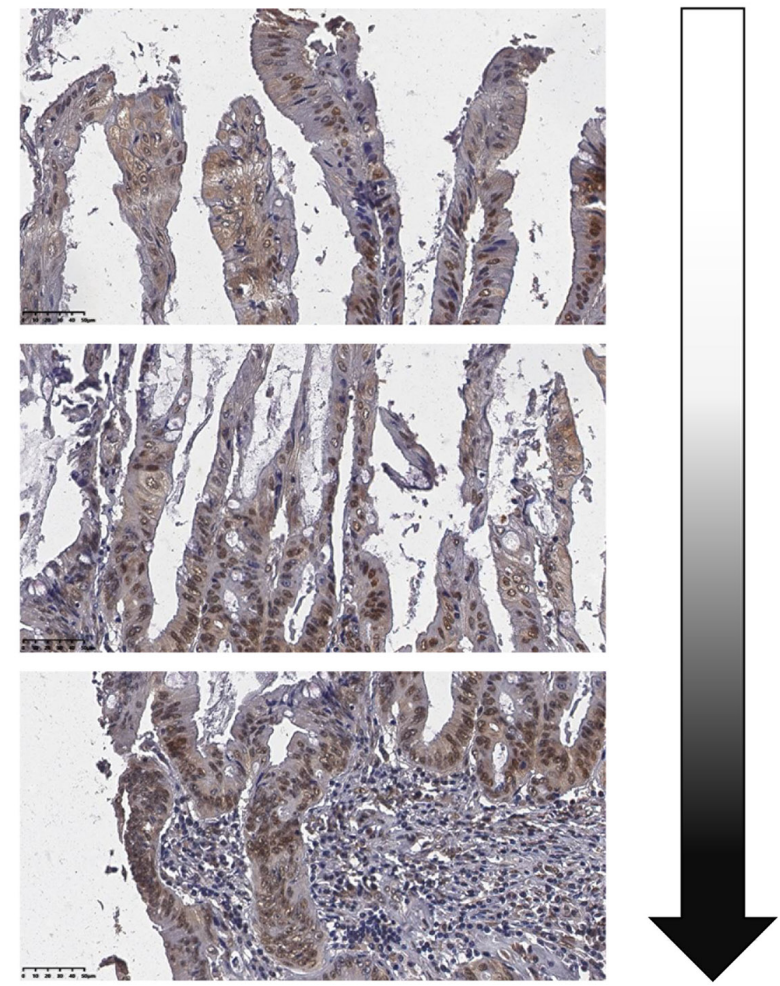

Figure 2. Heterogeneity of FOXK1 expression in one tumor. (A) Low expression of FOXK1 on the surface of the tumor. (B) High expression of FOXK1 at the bottom of the tumor. (C) High expression of FOXK1 in invasive tumor cells. Top panel magnification, x40; bottom panels magnification, x200 FOXK1, forkhead box K1.

analysis, the multivariate analysis using the Cox proportional hazards model showed that high FOXK1 expression was an independent predictor of tumor recurrence ( $\mathrm{P}=0.014$; Table II).

With respect to OS, the median for patients with resected $\mathrm{HC}$ was 16 months. However, patients with high FOXK1 expression in tumors had a significantly worse OS than those with low FOXK1 expression (14 months vs. 50 months; $\mathrm{P}<0.001$; Fig. 3B). Additionally, tumor invasion $(\mathrm{P}=0.014)$ and regional $\mathrm{LN}$ metastasis $(\mathrm{P}=0.033)$ also significantly influenced OS based on the univariate analysis (Table III). The multivariate analysis also showed that high FOXK1 expression was an independent prognostic factor $(\mathrm{P}=0.037$; Table III).

FOXK1 knockdown attenuates cell invasion and migration in vitro. To provide direct evidence for the critical role of FOXK1 in $\mathrm{HC}$, its expression was knocked down in $\mathrm{HC}$ cells in vitro using shRNA. HC cells exhibited high endogenous FOXK1
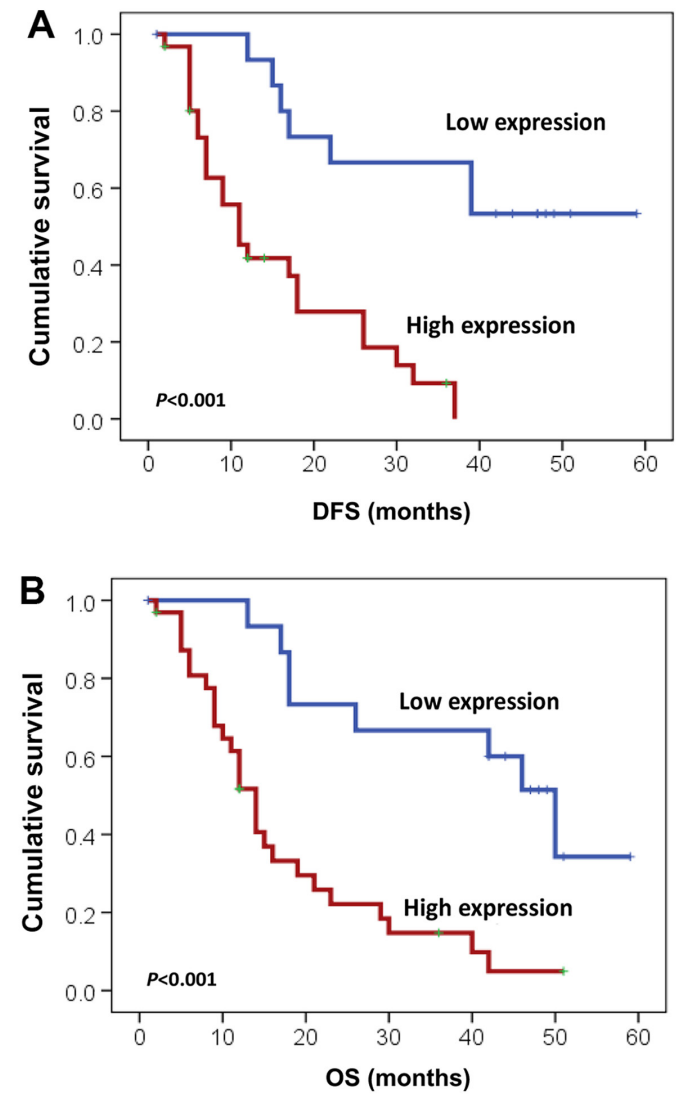

Figure 3. Kaplan-Meier curves of DFS and OS in patients with hilar cholangiocarcinoma. (A) DFS was significantly worse in patients with high FOXK1 expression in tumors than in those with low FOXK1 expression (11 vs. 42 months, $\mathrm{P}<0.001$ ). (B) OS was significantly worse in patients with high FOXK1 expression compared with those with low FOXK1 expression (14 vs. 50 months, $\mathrm{P}<0.001$ ). DFS, disease-free survival; OS, overall survival; FOXK1, forkhead box K1.

expression (Fig. 4A). Fig. 4B shows that the silencing of FOXK1 expression using shRNA was successful in RBE cells (Fig. 4B). Silencing endogenous FOXK1 did not affect cell proliferation (Fig. 4B), but it significantly inhibited cell invasion, as revealed by the Transwell assay ( $\mathrm{P}=0.0013$; Fig. 4C), and cell migration, as revealed by the wound healing assay (Fig. 4D). Western blotting revealed that FOXK1 knockdown resulted in the upregulation of E-cadherin and the downregulation of vimentin, MMP-9 and Twist (Fig. 4E).

FOXK1 knockdown reduces drug resistance in vitro. To explore chemoresistance in HC, FOXK1-knockdown RBE cells were treated with 5-FU and DDP. It was found that silencing endogenous FOXK1 expression in RBE cells increased their sensitivity to 5-FU and DDP (Fig. 5A). In addition, western blotting revealed that markers related to drug resistance, such as GST- $\pi$, MDR1 and P-gp, were downregulated in RBE cells in vitro when FOXK1 was suppressed (Fig. 5B).

\section{Discussion}

The present study determined the expression profile of FOXK1 in patients with $\mathrm{HC}$ and identified that it was considerably increased and significantly associated with tumor invasion and metastasis. However, it had no effect on the proliferation 
A
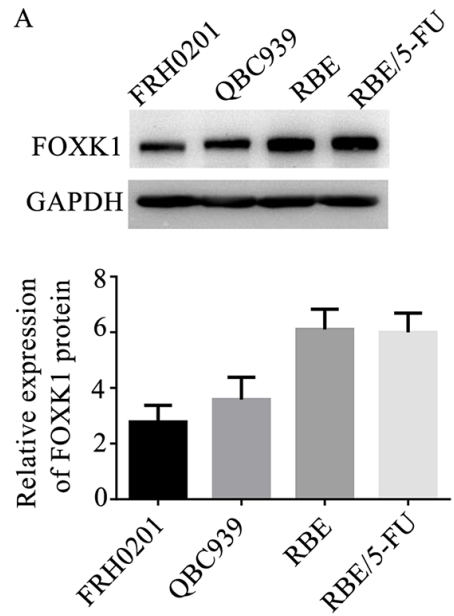

C RBE-shNC RBE-shFOXK1

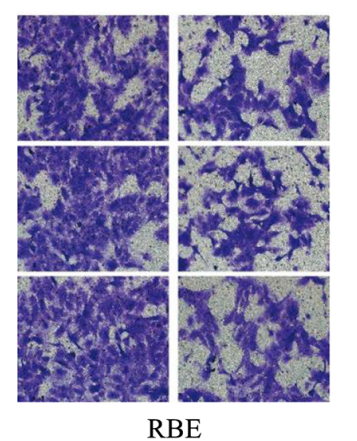

D RBE-shNC RBE-shFOXK1

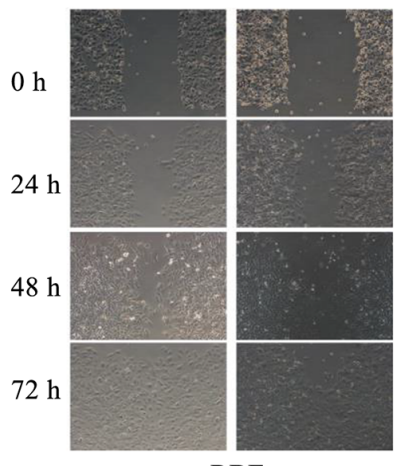

RBE

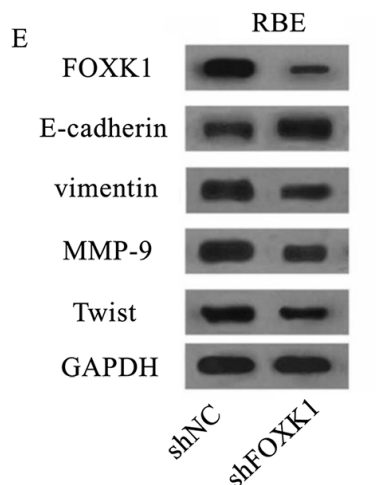

B
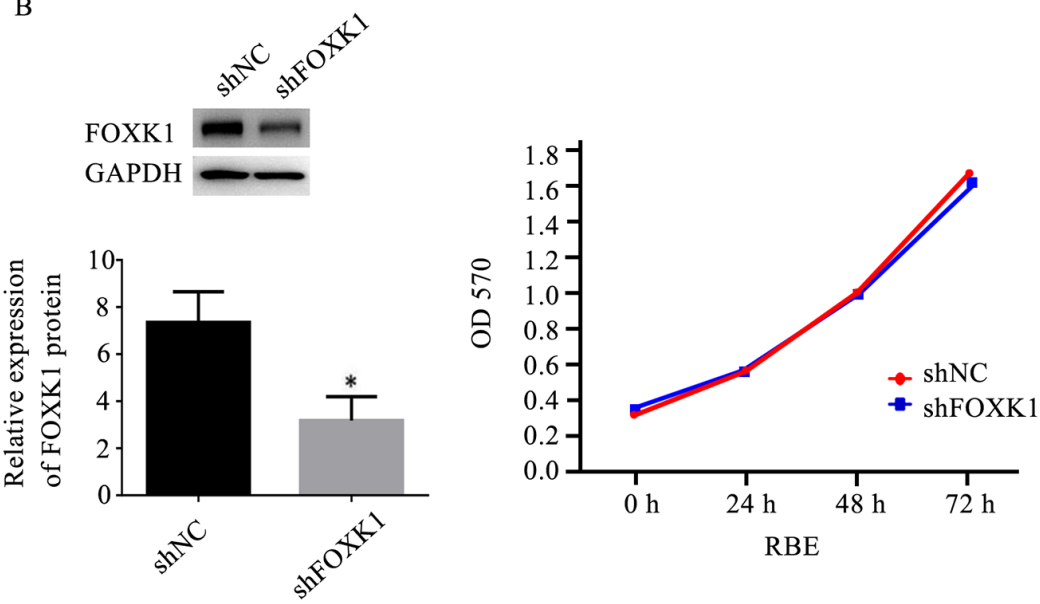

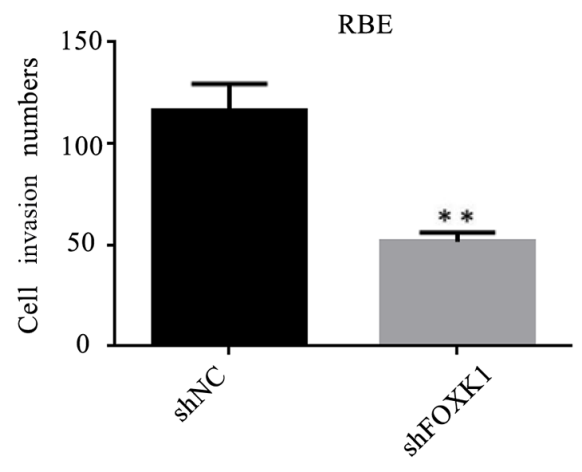

RBE
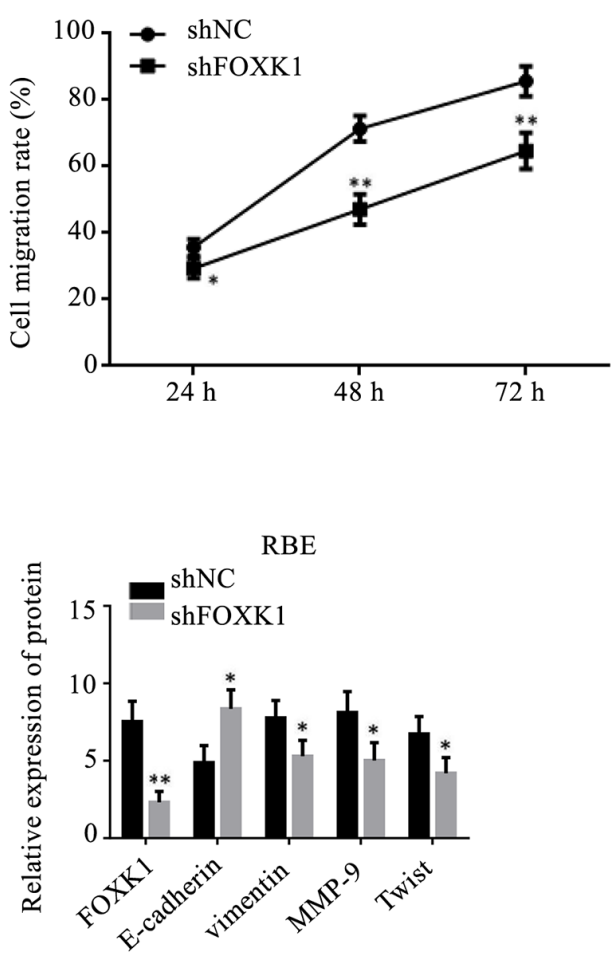

Figure 4. Inhibitory role of silencing FOXK1 on hilar cholangiocarcinoma cells. (A) Endogenous levels of FOXK1 in FRH0201, QBC939, RBE and RBE/5-FU cells. (B) RBE cells were infected with shFOXK1 or shNC and examined by western blotting. Cells infected with the shNC or shFOXK1 lentivirus were seeded into 96-well plates and cell growth was assessed by performing Cell Counting Kit-8 assays. (C) Transwell assay was performed to evaluate the effect of FOXK1 on cell invasion (magnification, x200) in RBE cells transfected with shFOXK1 compared with that in the sh-NC group. (D) Wound healing assay was used to evaluate the effect of FOXK1 on the migration (magnification, x200) of RBE cells transfected with shFOXK1 compared with the sh-NC group. (E) Levels of E-cadherin, vimentin, MMP-9 and Twist were measured by western blotting in FOXK1-silenced RBE cells. " $\mathrm{P}<0.05$, ${ }^{* * *} \mathrm{P}<0.01$ vs. shNC group. FOXK1, forkhead box K1; sh, short hairpin RNA; NC, negative control; MMP, metallopeptidase. 
A

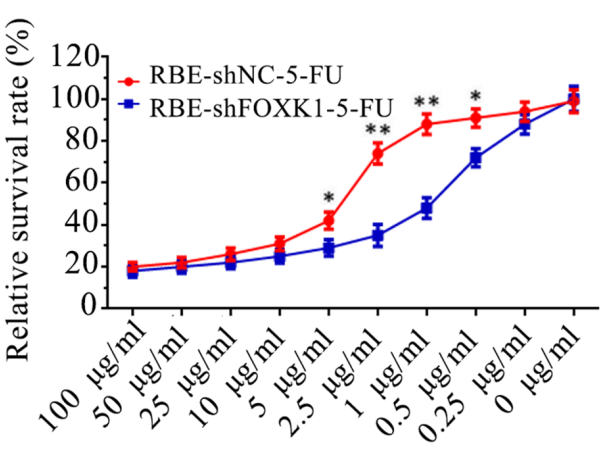

\begin{tabular}{|l|l|}
\hline & IC $_{50}$ \\
\hline RBE-shNC-5FU & $4.12 \mu \mathrm{M}$ \\
\hline RBE-shFOXK1-5-FU & $0.93 \mu \mathrm{M}$ \\
\hline
\end{tabular}
RBE-DDP

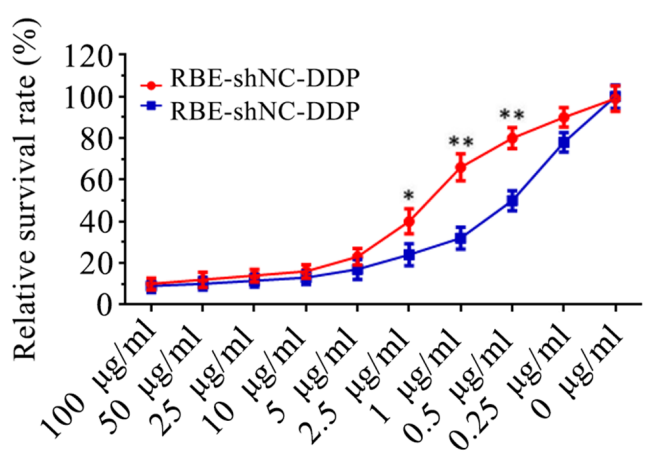

\begin{tabular}{|l|c|}
\hline & IC $_{50}$ \\
\hline RBE-shNC-DDP & $1.75 \mu \mathrm{g} / \mathrm{ml}$ \\
\hline RBE-shFOXK1-DDP & $0.52 \mu \mathrm{g} / \mathrm{ml}$ \\
\hline
\end{tabular}

B

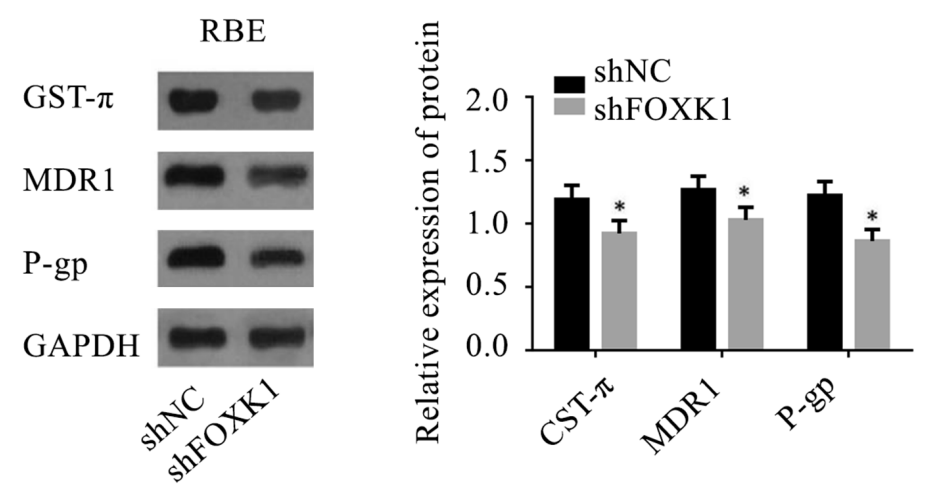

Figure 5. Role of FOXK1 in chemotherapy resistance of hilar cholangiocarcinoma cells. (A) RBE cells transfected with shFOXK1 have a lower IC 50 of 5-FU and DDP compared with the sh-NC group. (B) Levels of GST- $\pi$, MDR1 and P-gp were measured by western blotting in FOXK1-silenced RBE cells and the expression of GST- $\pi$, MDR1 and P-gp decreased in the shFOXK1 group compared with that in the sh-NC group. ${ }^{*} \mathrm{P}<0.05$, ${ }^{* *} \mathrm{P}<0.01$ vs. shNC group. FOXK1, forkhead box K1; sh, short hairpin RNA; 5-FU, 5-fluorouracil; DDP, cisplatin; GST, glutathione S-transferase; MDR1, multidrug resistance mutation 1; P-gp, P-glycoprotein; NC, negative control.

of HC. The data of survival follow-up revealed that its high expression was an independent predictor of tumor recurrence and $\mathrm{OS}$ after $\mathrm{HC}$ resection. However, the migratory and invasive abilities of RBE cells were inhibited and the expression of several epithelial-mesenchymal transition (EMT)-associated proteins were influenced following knockdown of FOXK1, which provided a novel molecular basis for the key role of FOXK1 in HC development and progression.

FOXK1 plays an oncogenic role in a number of solid tumors, and it has been observed to be upregulated in hepatocellular carcinoma, and gastric, colorectal, prostate, esophageal and ovarian cancer (7-11,15-17). This high expression of FOXK1 is also found to correlate significantly with malignant behaviors, including poor differentiation of esophageal cancer (10), size and metastasis of ovarian cancer (9), and differentiation, LN metastasis and AJCC stage of colorectal cancer (18). Consistent with these studies, the present study confirmed that FOXK1 was highly expressed in HC tissues, using resected $\mathrm{HC}$ specimens and matched specimens. High expression was identified to be associated with tumor invasion and metastasis. All these data supported the role of FOXK1 as an oncogene and suggested that it might serve as a potential therapeutic target for patients with $\mathrm{HC}$.
A number of clinicopathological variables have been identified as associated with tumor recurrence and OS for $\mathrm{HC}$, including tumor invasion, nerve invasion, regional LN metastasis, curative resection and disease progression (19-21). In the small HC cohort of the present study, tumor invasion and LN metastasis significantly affected disease progression and OS. However, these factors did not independently predict tumor recurrence and patient outcome. Possible reasons for this discrepancy are the limited number of subjects in the cohort and the fact that this cohort involved a number of patients with $\mathrm{HC}$ with advanced-stage disease. A number of studies have introduced biological markers into the Cox regression model, including amino acid transporter A1, proline-rich protein 11, pyruvate kinase PKM and Annexin A1 (12,13,22-24). The survival analysis from the present study showed that high FOXK1 expression in tumors was associated with shorter PFS and worse outcome. In addition, FOXK1 was an independent predictor of tumor recurrence and OS in patients with $\mathrm{HC}$. These findings suggested that this protein might be a potential biomarker to predict disease progression and outcome in patients with $\mathrm{HC}$.

Locally advanced or metastatic $\mathrm{HC}$ at diagnosis makes patients ineligible for surgical resection and thus limits their 
overall 5-year survival. Given the association between high FOXK1 expression and tumor invasion or LN metastasis, the present study aimed to detect the role of FOXK1 in cell migration by suppressing its expression and exploring a possible associated mechanism. The present study used shRNA interference transfection to construct an effective FOXK1-knockdown cell line. It was identified that silencing endogenous FOXK1 significantly inhibited HC cell migration and invasion, as revealed by the Transwell and wound healing assays. Overall, the data indicated that FOXK1 played a critical role in disease progression. Several previous studies have revealed that EMT confers properties that are critical for invasion and distant metastasis to neoplastic epithelial cells $(25,26)$. It has been demonstrated that the knockdown of FOXK1 inhibits transforming growth factor $\beta$-induced EMT (27). Its overexpression induces this process by upregulating cysteine-rich angiogenic inducer 61 in colorectal cancer (7), whereas knockdown prevents an EMT phenotype through the upregulation of E-cadherin and downregulation of $\mathrm{N}$-cadherin in prostate cancer cells (28). Therefore, the present study determined the expression of EMT-related proteins, such as E-cadherin, vimentin, MMP-9 and Twist, by western blotting in FOXK1-knockdown cell lines. The loss of E-cadherin and the upregulation of vimentin and Twist represent the EMT process, while MMP-9 is a key component that mediates cell adhesion. According to previous studies $(7,25-28)$ and the results of the present study, FOXK1 could be a critical inducer of EMT and it is proposed that FOXK1 plays a critical oncogenic role by promoting the EMT process. Although further research is needed to explore the molecular mechanisms, the results of the present study provided a novel molecular basis for the key role of FOXK1 in HC development and progression.

In addition to the lack of effective biomarkers, chemoresistance is also an important factor in the high mortality of HC. Silencing FOXK1 increased sensitivity to 5-FU and DDP, and downregulated the expression of GST- $\pi$, MDR1 and P-gp, which are related to drug resistance, in $\mathrm{RBE}$ cells in vitro. Thus, FOXK1 may serve as a putative target for HC.

In summary, FOXK1 was highly expressed in $\mathrm{HC}$ and associated with tumor invasion and LN metastasis in Chinese patients with HC. Furthermore, high FOXK1 expression was an independent predictor of tumor recurrence and OS. Thus, these results indicated that FOXK1 can facilitate cancer metastasis by regulating EMT-associated proteins in $\mathrm{HC}$ and plays a role in chemoresistance.

\section{Acknowledgements}

The authors would like to acknowledge Dr Wenlong Yu from the Eastern Hepatobiliary Hospital (Shanghai, China) and Dr Ying Chen from Changhai Hospital (Shanghai, China) for providing HC samples.

\section{Funding}

The present study was partially supported by Beijing Municipal Administration of Hospitals Clinical Medicine Development of Special Funding Support (grant no. ZYLX201504) and National Key Technologies R\&D Program (grant no. 2015BAI13B09)

\section{Availability of data and materials}

The datasets used and/or analyzed during the current study are available from the corresponding author on reasonable request.

\section{Authors' contributions}

$\mathrm{ZZ}$ conceived and designed the study. YF and ZB acquired the data. YF, ZB and JS were responsible for data analysis and interpretation. YF and ZZ wrote the manuscript. All authors read and approved the final manuscript.

\section{Ethics approval and consent to participate}

The tissue sample experiments were approved by the Ethics Committee of The Affiliated Hospital of Qingdao University. Informed consent written from all participants (or their parent or legal guardian in the case of children under 16) was obtained to participate in the study or to use their tissues.

\section{Patient consent for publication}

Not applicable.

\section{Competing interests}

The authors declare that they have no competing interests.

\section{References}

1. Bray F, Ferlay J, Soerjomataram I, Siegel RL, Torre LA and Jemal A: Global cancer statistics 2018: GLOBOCAN estimates of incidence and mortality worldwide for 36 cancers in 185 countries. CA Cancer J Clin 68: 394-424, 2018.

2. Poruk KE, Pawlik TM and Weiss MJ: Perioperative management of hilar cholangiocarcinoma. J Gastrointest Surg 19: 1889-1899, 2015.

3. Donati M, Stang A, Stavrou GA, Basile F and Oldhafer KJ: Extending resectability of hilar cholangiocarcinomas: How can it be assessed and improved? Future Oncol 15: 193-205, 2019.

4. Lewis HL, Rahnemai-Azar AA, Dillhoff M, Schmidt CR and Pawlik TM: Current management of perihilar cholangiocarcinoma and future perspectives. Chirurgia (Bucur) 112: 193-207, 2017.

5. Wu Y, Peng Y, Wu M, Zhang W, Zhang M, Xie R, Zhang P, Bai Y, Zhao J, Li A, et al: Oncogene FOXK1 enhances invasion of colorectal carcinoma by inducing epithelial-mesenchymal transition. Oncotarget 7: 51150-51162, 2016.

6. Shi X, Wallis AM, Gerard RD, Voelker KA, Grange RW, DePinho RA, Garry MG and Garry DJ: Foxk1 promotes cell proliferation and represses myogenic differentiation by regulating Foxo4 and Mef2. J Cell Sci 125: 5329-5337, 2012.

7. Huang X, Xiang L, Li Y, Zhao Y, Zhu H, Xiao Y, Liu M, Wu X, Wang Z, Jiang P, et al: Snail/FOXK1/Cyr61 signaling axis regulates the epithelial-mesenchymal transition and metastasis in colorectal cancer. Cell Physiol Biochem 47: 590-603, 2018.

8. Chen F, Xiong W, Dou K and Ran Q: Knockdown of FOXK1 Suppresses proliferation, migration, and invasion in prostate cancer cells. Oncol Res 25: 1261-1267, 2017.

9. Li P, Yu Z, He L, Zhou D, Xie S, Hou H and Geng X: Knockdown of FOXK1 inhibited the proliferation, migration and invasion in hepatocellular carcinoma cells. Biomed Pharmacother 92: 270-276, 2017.

10. Chen D, Wang K, Li X, Jiang M, Ni L, Xu B, Chu Y, Wang W, Wang H, Kang H, et al: FOXK1 plays an oncogenic role in the development of esophageal cancer. Biochem Biophys Res Commun 494: 88-94, 2017. 
11. Li L, Gong M, Zhao Y, Zhao X and Li Q: FOXK1 facilitates cell proliferation through regulating the expression of $\mathrm{p} 21$, and promotes metastasis in ovarian cancer. Oncotarget 8: 70441-70451, 2017.

12. Yu G, Yu W, Jin G, Xu D, Chen Y, Xia T, Yu A, Fang W, Zhang X, Li Z, et al: PKM2 regulates neural invasion of and predicts poor prognosis for human hilar cholangiocarcinoma. Mol Cancer 14: 193, 2015.

13. Jiao X, Yu W, Qian J, Chen Y, Wei P, Fang W and Yu G: ADAM-17 is a poor prognostic indicator for patients with hilar cholangiocarcinoma and is regulated by FoxM1. BMC Cancer 18: 570, 2018.

14. Fang W, Cui H, Yu D, Chen Y, Wang J and Yu G: Increased expression of phospho-acetyl-CoA carboxylase protein is an independent prognostic factor for human gastric cancer without lymph node metastasis. Med Oncol 31: 15, 2014

15. Wu Y, Xie R, Liu X, Wang J, Peng Y, Tang W, Wu M, Zhang P, Ba Y, Zhao J, et al: Knockdown of FOXK1 alone or in combination with apoptosis-inducing 5-FU inhibits cell growth in colorectal cancer. Oncol Rep 36: 2151-2159, 2016.

16. Xie R, Wang J, Liu X, Wu L, Zhang H, Tang W, Li Y, Xiang L, Peng Y, Huang X, et al: RUFY3 interaction with FOXK1 promotes invasion and metastasis in colorectal cancer. Sci Rep 7: 3709, 2017

17. Zhang P, Tang WM, Zhang H, Li YQ, Peng Y, Wang J, Liu GN, Huang XT, Zhao JJ, Li G, et al: MiR-646 inhibited cell proliferation and EMT-induced metastasis by targeting FOXK1 in gastric cancer. Br J Cancer 117: 525-534, 2017.

18. Wu M, Wang J, Tang W, Zhan X, Li Y, Peng Y, Huang X, Bai Y, Zhao J, Li A, et al: FOXK1 interaction with FHL2 promotes proliferation, invasion and metastasis in colorectal cancer. Oncogenesis 5: e271, 2016.

19. Razumilava $\mathrm{N}$ and Gores GJ: Cholangiocarcinoma. Lancet 383 : $2168-2179,2014$
20. Bergquist A and von Seth E: Epidemiology of cholangiocarPlentz RR and Malek NP: Clinical presentation, risk factors and staging systems of cholangiocarcinoma. Best Pract Res Clin Gastroenterol 29: 245-252, 2015.

21. Plentz RR and Malek NP: Clinical presentation, risk factors and staging systems of cholangiocarcinoma. Best Pract Res Clin Gastroenterol 29: 245-252, 2015.

22. Wang D, Zhang H, Fang Z and Yu G: Annexin-1 downregulation is associated with clinical outcome in Chinese patients with hilar cholangiocarcinoma. Eur Surg Res 45: 151-157, 2010.

23. Yu WL, Cong WM, Zhang Y, Chen Y, Wang F and Yu G: Overexpression of ATA1/SLC38A1 predicts future recurrence and death in Chinese patients with hilar cholangiocarcinoma. J Surg Res 171: 663-668, 2011.

24. Chen Y, Cha Z, Fang W, Qian B, Yu W, Li W, Yu G and Gao Y: The prognostic potential and oncogenic effects of PRR11 expression in hilar cholangiocarcinoma. Oncotarget 6: 20419-20433, 2015.

25. Lambert AW, Pattabiraman DR and Weinberg RA: Emerging biological principles of metastasis. Cell 168: 670-691, 2017.

26. Chen S, Kang X, Liu G, Zhang B, Hu X and Feng Y: a7-Nicotinic acetylcholine receptor promotes cholangiocarcinoma progression and epithelial-mesenchymal transition process. Dig Dis Sci 64: 2843-2853, 2019

27. Zhang H, Wu X, Xiao Y, Wu L, Peng Y, Tang W, Liu G, Sun Y, Wang J, Zhu H, et al: Coexpression of FOXK1 and vimentin promotes EMT, migration, and invasion in gastric cancer cells. J Mol Med (Berl) 97: 163-176, 2019.

28. Wang J, Liu G, Liu M, Xiang L, Xiao Y, Zhu H, Wu X, Peng Y, Zhang W, Jiang P, et al: The FOXK1-CCDC43 axis promotes the invasion and metastasis of colorectal cancer cells. Cell Physiol Biochem 51: 2547-2563, 2018.

This work is licensed under a Creative Commons Attribution-NonCommercial-NoDerivatives 4.0 International (CC BY-NC-ND 4.0) License. 